\title{
Shear and compression behaviour of sheet moulding compounds
}

\author{
Steven Le Corre ${ }^{\mathrm{a}}$, Laurent Orgéas ${ }^{\mathrm{a}}$, Denis Favier ${ }^{\mathrm{a}}$, Ali Tourabi ${ }^{\mathrm{a}}$, \\ Abderrahim Maazouz ${ }^{\mathrm{b}}$, Cécile Venet ${ }^{\mathrm{c}}$ \\ ${ }^{a}$ Laboratory Soils Solids Structures (L3S), UMR CNRS 5521, University Joseph Fourier, INPG, BP 53, 38041, Grenoble Cedex 9 , France \\ ' INSA Lyon, UMR CNRS 5627, Domaine Universitaire de la Doua, 69000 Villeurbanne, France \\ ${ }^{c}$ Schneider Electric Industries, Corporate Research Division, Analytics Group, A2 plant, rue Volta F 38050 Grenoble cedex 9, France
}

\begin{abstract}
At the present time, the rheology of sheet moulding compounds (SMC) during forming is not well known. In order to provide better experimental data, an experimental program was carried out as a result of the development of a new rheometer especially dedicated to this kind of material. Homogeneous simple compression and simple shear tests are presented. They allow the evalua tion of the influence of the main parameters on the SMC behaviour: strain rate, temperature and fibre fraction. It is shown that the $\mathrm{SMC}$ can be considered as a strongly anisotropic non linear viscous medium.
\end{abstract}

Keywords: A. Short fibre composites; B. Non linear behaviour; B. Thermomechanical properties; C. Anisotropy; Compression moulding

\section{Introduction}

Sheet molding compounds (SMC) refer to a family of composites made from a thermoset polymer matrix reinforced by short glass fibres around $25 \mathrm{~mm}$ long. SMC are manufactured as $2-3 \mathrm{~mm}$ thick pre-impregnated sheets in which fibres are randomly distributed. Following cutting and the creation of a stack consisting of a set of layers (3-10), SMC sheets are formed by compression in a mould heated at $423 \mathrm{~K}$, allowing rapid curing of the part. These materials are mainly used in the automotive industry for production of car body parts and semi-structural parts as well as in electrical engineering to make large-size enclosures and cabinets.

To date, a large amount of work has concentrated on analysis of SMC flow in compression, based mainly on experiments reproducing real forming conditions $[1,2]$. This work has generated a certain number of simplified flow models, now extensively used by industrial simulation tools [3-5]. Although process modelling literature is plentiful, material rheological characterisation studies are relatively rare and usually incomplete [6-8]. This is because conventional rheometry tools are poorly adapted to this type of material which requires large samples in relation to fibre size and thus high loading forces. The recent studies conducted by [9-11] come closer, however, to the concerns of our research work.

The aim of this paper is to complete the material database available in the literature. Therefore, experimental characterisation of the thermomechanical behaviour of SMC containing a variety of fibre fractions was performed under homogeneous mechanical loading close to those encountered during the process. For this purpose, a rheometer particularly dedicated to the study of this material type has been developed. This rheometer has allowed us to conduct simple compression and shear tests at strain rates and temperatures representative of the process.

\section{Experimental device: annular rheometer}

The large size and arrangement of the fibres in SMC sheets make conventional rheometer tools unsuitable for characterising SMC mechanical behaviour. A specific large-size rheometer has therefore been developed (Fig. 1). Mounted on an MTS tension/torsion hydraulic testing machine, it performs simple compression and 


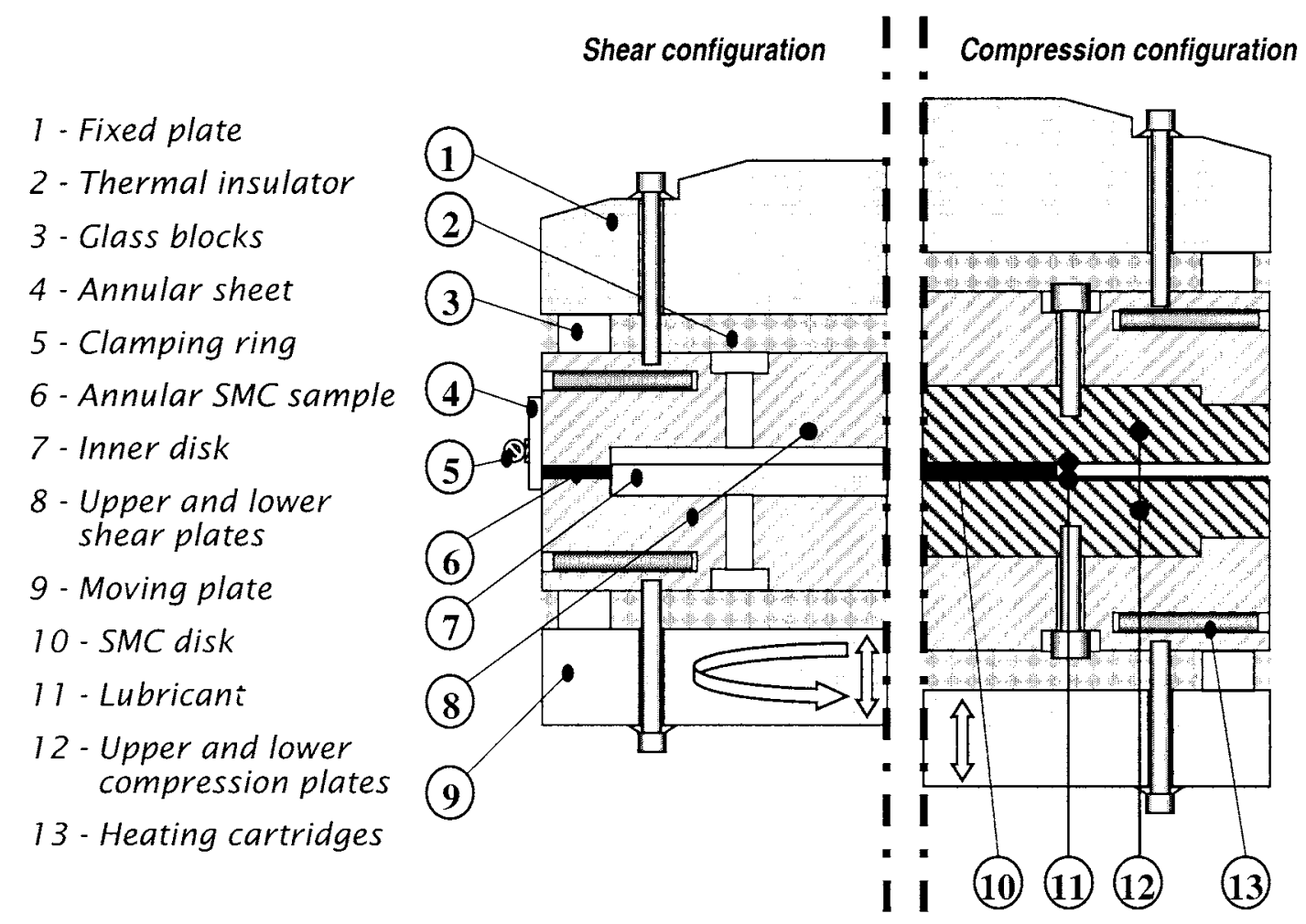

Fig. 1. Shear compression rheometer.

shear tests at strain rates and temperatures representative of the process. The press actually allows a maximum axial force of $10 \mathrm{kN}$ at a maximum axial displacement velocity of $1 \mathrm{~ms}-1$ and a maximum torque of $1.2 \mathrm{kN} \mathrm{m}$ at a maximum angular velocity of $1000^{\circ}$ $\mathrm{s}^{-1}$. Furthermore, a resistor-based heating system allows temperature regulation up to $573 \mathrm{~K}$ at $\pm 0.5 \mathrm{~K}$, as shown in Fig. 1.

\section{Material and experimental procedure}

Experiments were performed on standard SMC sheets of the Low Profile type produced by Mecelec Composites et Recyclage (Tournon, France). The fibre-free sheet composition (paste) is given in Table 1. Tested fibre-volume fractions $f^{\mathrm{f}}$ are $0,3.6,7.3,11.2,15.1,19.2$ and $23.4 \%$. As shown in Table 1, a large amount of inhibitor was added compared with the conventional

Table 1

Composition of the paste

\begin{tabular}{lclc}
\hline Component & $\begin{array}{l}\text { Mass } \\
\text { fraction (\%) }\end{array}$ & Component & \multicolumn{1}{c}{$\begin{array}{l}\text { Mass } \\
\text { fraction (\%) }\end{array}$} \\
\hline Polyester resin & 19.6 & M additive & 0.76 \\
Styrene & 1.56 & LP additive & 13.29 \\
Catalisor + inhibitor & 0.56 & MgO & 0.85 \\
ZnSt & 1.31 & $\mathrm{CaCO} 3$ & 62.07 \\
\hline
\end{tabular}

formulation in order to allow isothermal tests at high temperature without chemical reaction: to perform a homogeneous test at high temperature, a heating time of approximately $10 \mathrm{~min}$ is required.

In the "compression" configuration, the rheometer allows simple compression tests to be performed on disks of initial diameter $\phi_{0}$ ranging from 50 to $200 \mathrm{~mm}$ and initial height $h_{0}$ ranging from 6 to $9 \mathrm{~mm}$. The upper and lower faces of the samples and the surface of the compression plates are thoroughly lubricated before each test to obtain homogeneous simple compression kinematics. Preliminary systematic marking of samples ensures that the resulting kinematics is correct. The material is deformed at a constant axial strain rate $\dot{\varepsilon}=$ $\dot{h} / h$ and at a constant temperature T. Tested strain rates cover the range $10^{-3}-10 \mathrm{~s}^{-1}$ for temperatures of 296 (noted $T_{0}$ ), 313, 333 and $353 \mathrm{~K}$. To analyse the results, the logarithmic strain $\varepsilon=\ln \left(h_{0} / h\right)$ was used, where $h$ and $h_{0}$ are, respectively, the current height and the initial height of the sample. The axial stress $\sigma$ was calculated from measurement of axial force $F$ assuming incompressibility, which allows the use of the initial surface of the sample $S_{0}=\frac{\pi}{4} \phi_{0}^{2}$

$\sigma=\frac{F}{S}=\frac{F}{S_{0}} \frac{h}{h_{0}}$

Typical SMC response to a constant strain rate $\dot{\varepsilon}$ is shown in Fig. 2 which summarises a series of tests 
performed on four different samples with the same fibre fraction $f^{\mathrm{f}}=11.2 \%$ and at $\dot{\varepsilon}=10^{-1} \mathrm{~s}^{-1}$, interrupted at different strain states. This figure first verifies that an homogeneous simple compression kinematics is properly complied with up to more than 0.5 of logarithmic strain (see sample marking). It also provides the results dispersion obtained on this industrial material, which is around $\pm 10 \%$. In a strain range representative of the process $(\varepsilon \leqslant 1.3)$, SMC response exhibits the characteristic shape given by curve D in Fig. 2. Regardless of $f^{\mathrm{f}}$, $T$ and $\dot{\varepsilon}$, we systematically observe a first rapid increase in stress which is followed by a plateau characterised by a threshold stress $\sigma_{\text {threshold }}$, starting at roughly $\varepsilon=0.25$.

In the "shear" configuration, the rheometer shears SMC rings with an external diameter $\phi_{\text {ext }}$ of $400 \mathrm{~mm}$, an internal diameter $\phi_{\text {int }}$ of $320 \mathrm{~mm}$ and a height $h$ of 2 to 4 $\mathrm{mm}$ corresponding to a single SMC layer. Adherence of SMC to the walls is ensured by inserting a thin and highly resistant adhesive film between the sample and the plates. Systematic side marking of the rings highlights the appearance of any slippage. To analyse the results, observing that $\phi_{\text {ext }}$ is large compared with width $\frac{\phi_{\text {ext }} \phi_{\text {int }}}{2}$, the shear strain rate is approximated by:

$\dot{\gamma}=\frac{\Omega \cdot R_{\mathrm{m}}}{h}=\frac{\Omega}{h} \cdot \frac{\phi_{\mathrm{ext}}+\phi_{\text {int }}}{2}$

where $\Omega$ is the angular velocity of the plate and $R_{\mathrm{m}}$ the mean radius of the sample. The rings are sheared at constant $\Omega$ and thus at constant shear rate $\dot{\gamma}$. As in compression, $\dot{\gamma}$ covers the range $10^{-3}-10 \mathrm{~s}^{-1}$ and tested temperatures are 296,313 and $333 \mathrm{~K}$. At last, it is worth noting that the device enables to apply a normal force during shearing to get closer to industrial moulding conditions and to improve contact conditions.

The characteristic SMC response at constant $\dot{\gamma}$ is given in Fig. 3 which presents snapshots taken for different deformations during a shear test on a paste ring. This figure verifies that simple shear remains homogeneous up to a shear deformation $\dot{\gamma}$ of more than 1 . The shape of the response, presented on the paste, is similar regardless of $f^{\mathrm{f}}, T$ and $\dot{\gamma}$. First there is a rapid increase in stress which peaks around $\gamma=1$. This peak is then followed by a slow drop in stress that continues beyond a strain of 4 .

\section{Paste behaviour}

Fig. 4 shows in a graph equivalent shear stress $\tau_{\mathrm{eq}}$ (in the sense of Von Mises)/equivalent shear strain $\gamma_{\mathrm{eq}}$ (in the sense of Von Mises), a simple compression test and a shear test performed on the paste at room temperature and at an equivalent shear rate $\dot{\gamma}_{\text {eq }}$ of $0.173 \mathrm{~s}^{-1}$. This figure shows that over a strain range such that $\gamma_{\mathrm{eq}}<1$, paste behaves like a Von Mises material. This figure also shows that paste behaviour depends on the strain state. However, in what follows, to analyse the effect of other variables such as temperature and strain rate, results are analysed at an equivalent strain of 1 .

Fig. 5 shows the effect of strain rate on the equivalent viscosity of paste defined by $\eta_{\mathrm{eq}}^{\mathrm{p}}=\cdot \frac{\tau_{\mathrm{eq}}}{\grave{\gamma}_{\mathrm{eq}}}$, for the tests performed at $T=296 \mathrm{~K}$. Regardless of the type of loading, $\eta_{\mathrm{eq}}^{\mathrm{p}}$ is a power-law function of $\dot{\gamma}$ whose strain rate sensitivity $n^{\mathrm{p}}$ is independent of $T$ (in the tested temperature range):

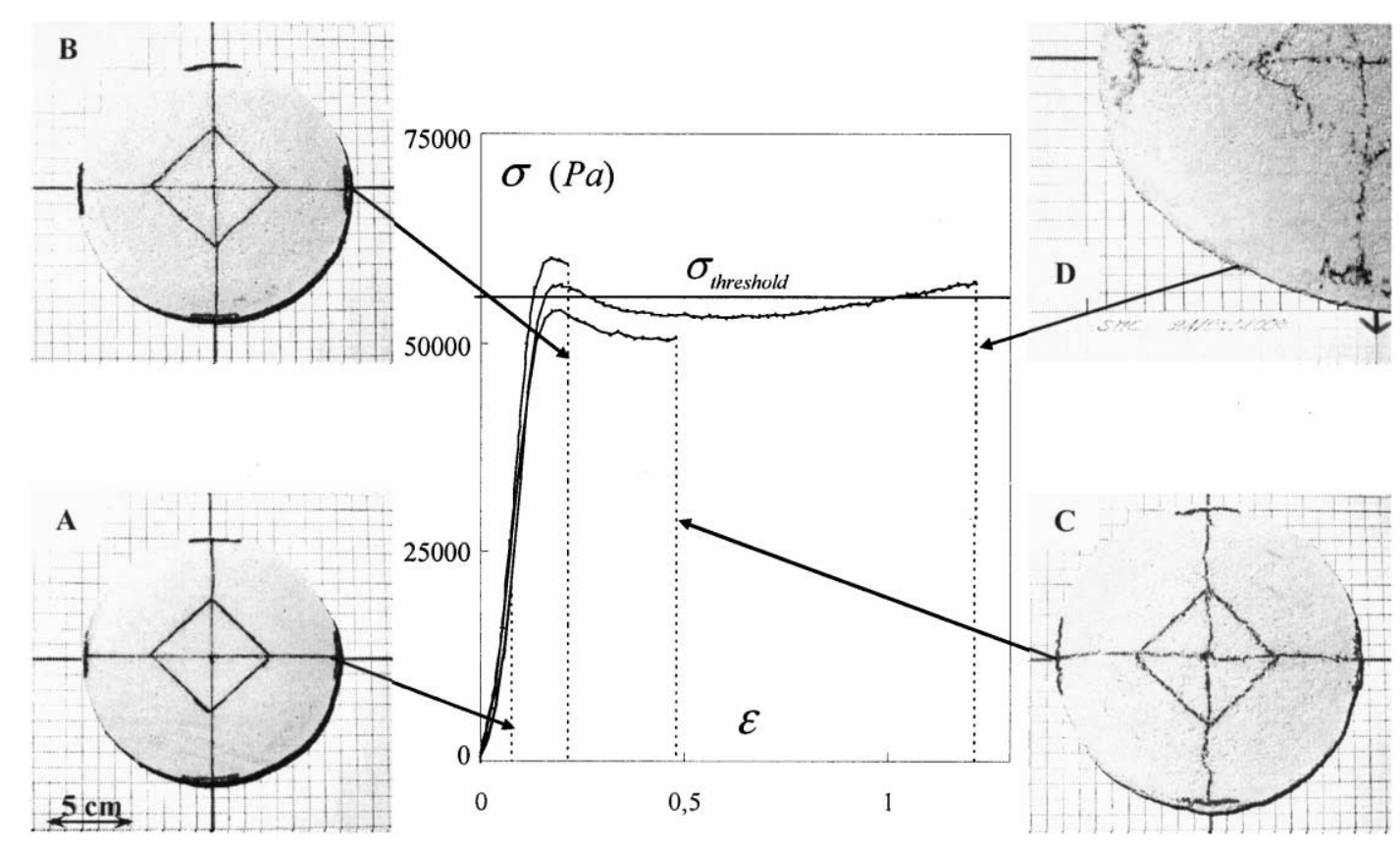

Fig. 2. Interrupted compression tests at $\varepsilon \quad 0.075(\mathrm{~A}), 0.21(\mathrm{~B}), 0.48(\mathrm{C})$ and $1.2(\mathrm{D}), \dot{\varepsilon} \quad 0.1 \mathrm{~s}^{1}, T \quad 296 \mathrm{~K}, f^{\mathrm{f}} \quad 11.2 \%$. 
B

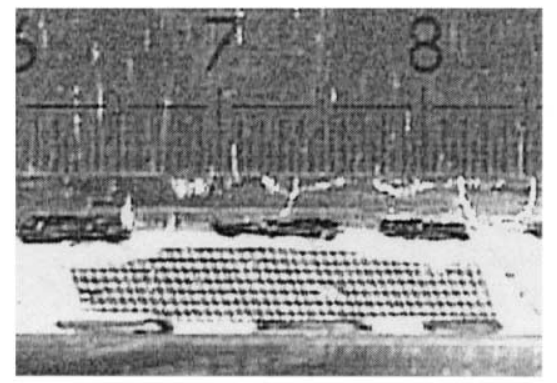

$\mathrm{C}$

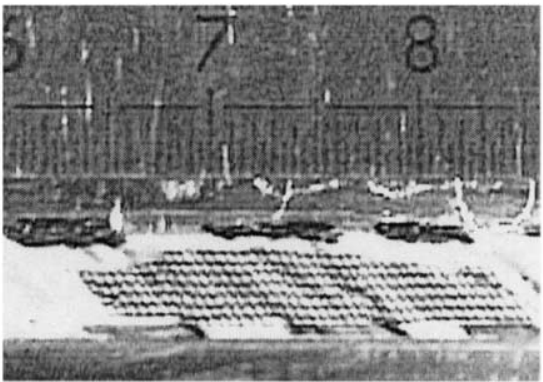

15000

$\tau(P a)$

A

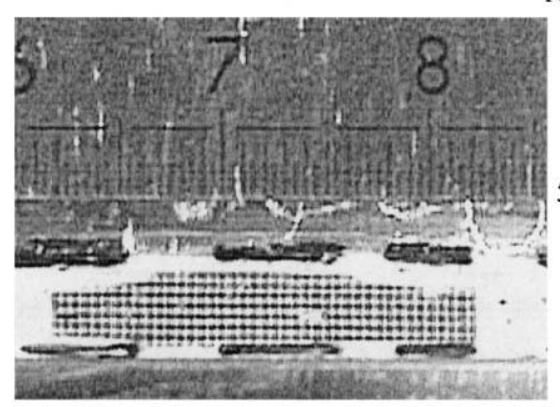

10000

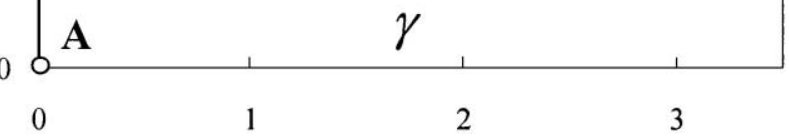

Fig. 3. Shear test on the paste at $\dot{\gamma} \quad 0.1 \mathrm{~s}^{1}$ and $T \quad 296 \mathrm{~K}$ : homogeneity of the deformation.

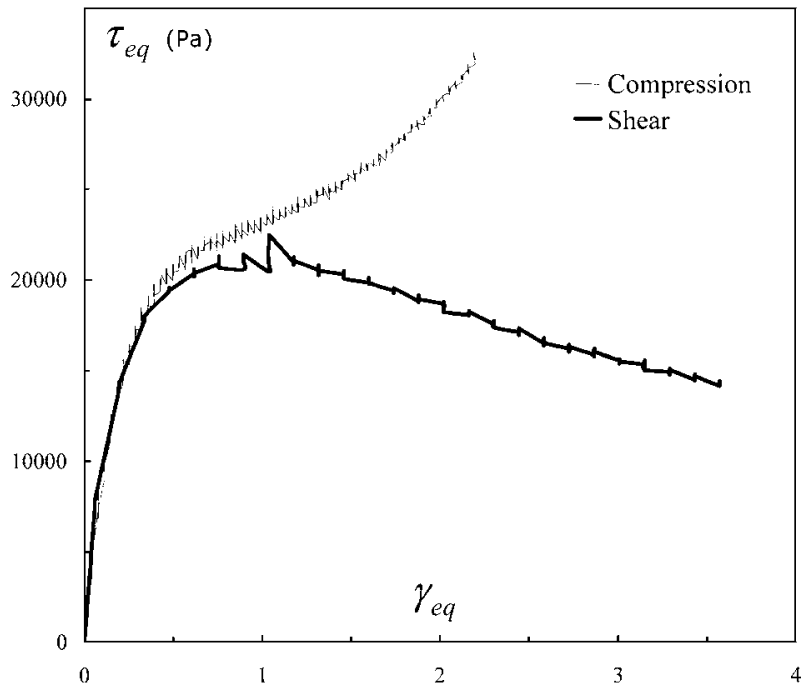

Fig. 4. Compression and shear tests on paste at room temperature, $\dot{\gamma}_{\mathrm{eq}} 0.173 \mathrm{~s}^{1}$.

$\eta_{e q}^{p}=\eta_{1}(T) \cdot\left(\frac{\dot{\gamma}_{\mathrm{eq}}}{\dot{\gamma}_{0}}\right)^{n^{\mathrm{p}} 1}$

where $n^{\mathrm{p}}=0.58$, and $\dot{\gamma}_{0}=1 \mathrm{~s}^{1}$

The temperature effect is then determined by plotting evolution of reduced viscosity $\eta_{\mathrm{p}}^{\star}=\eta_{\mathrm{eq}}^{\mathrm{p}} / \eta_{0}\left(\dot{\gamma}_{\mathrm{eq}} / \dot{\gamma}\right)^{n^{\mathrm{p}}}$ with temperature (Fig. 6). An Arrhenius type relation was used to fit experimental data:

$\eta_{1}(T)=\eta_{0} \exp \left(-b\left(1 / T_{0}-1 / T\right)\right)$

with $\eta_{0}=55000 \mathrm{~Pa} \mathrm{~s}, T_{0}=296 \mathrm{~K}$ and $b=4900 \mathrm{~K}$.

\section{SMC compression behaviour}

Fibre size is a characteristic length of the SMC. Thus, to experimentally characterise their behaviour, samples size must be sufficiently large with respect to this internal length. This has been verified on a SMC with $19 \%$ of fibre tested at $T_{0}$ and $\dot{\varepsilon}=10^{-2} \mathrm{~s}^{-1}$. Tests conducted on various initial diameters showed that for $\phi \geqslant 100$ $\mathrm{mm}$, the recorded response became stable and reproducible. Henceforward, $160 \mathrm{~mm}$ diameter samples were systematically used.

The effect of $T$ and $\dot{\varepsilon}$ on material response was analysed by means of SMC compression viscosity, defined by: $\eta_{\text {comp }}^{\text {smc }}=\frac{\sigma_{\text {threshold }}}{\dot{\varepsilon}}$. Fig. 7 illustrates the influence of $\dot{\varepsilon}$ at room temperature and for various fibre fractions. The first noteworthy result is that $\eta_{\mathrm{comp}}^{\mathrm{smc}}$ follows a power-law similar to that of paste with, however, a slightly lower 
strain rate sensitivity $n^{\mathrm{s}}=0.45$, regardless of fibre fraction. Only the consistency $\eta_{1, \text { comp }}^{\text {smc }}$ varies with fibre fraction.

Furthermore, we can observe that this result is true irrespective of temperature (Fig. 8), showing that SMC strain rate sensitivity $n^{\mathrm{s}}$ neither depends on $T$ nor on $f^{\mathrm{f}}$ and lets us produce the equation:

$\eta_{\mathrm{comp}}^{\mathrm{smc}}=\eta_{1, \mathrm{comp}}^{\mathrm{smc}}\left(T, f^{\mathrm{f}}\right)\left(\frac{\dot{\varepsilon}}{\dot{\varepsilon}_{0}}\right)^{n^{\mathrm{s}}} 1$, with $\dot{\varepsilon}_{0}=1 \mathrm{~s}{ }^{1}$

Fig. 9 shows the evolution as a function of $f^{\mathrm{f}}$ at room temperature of reduced viscosity $\eta_{\text {comp }}^{\star}$, defined by the ratio:

$\eta_{\text {comp }}^{\star}=\frac{\eta_{1, \mathrm{comp}}^{\mathrm{smc}}\left(T, f^{\mathrm{f}}\right)}{3 \eta_{1}(T)}$

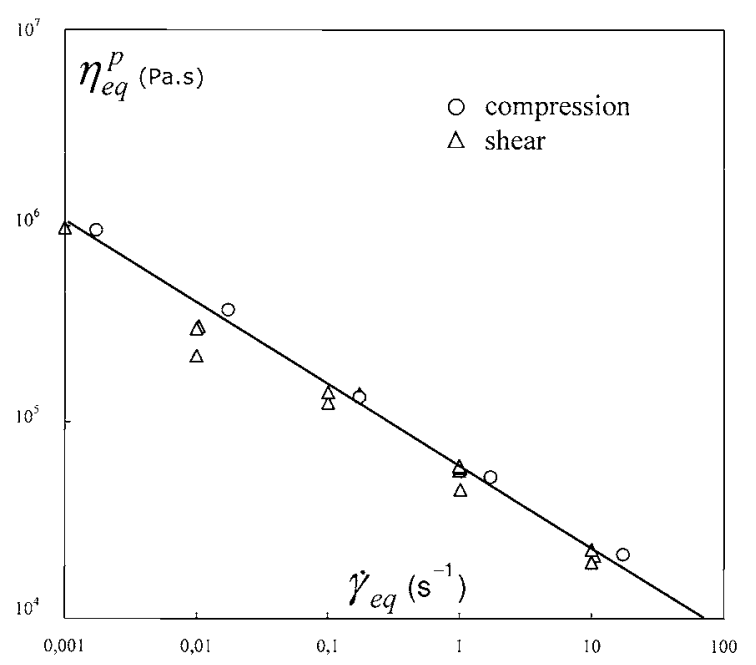

Fig. 5. Influence of $\dot{\gamma}_{\mathrm{eq}}$ on paste equivalent viscosity $\eta_{\mathrm{eq}}^{\mathrm{p}}$ at $T 296 \mathrm{~K}$.

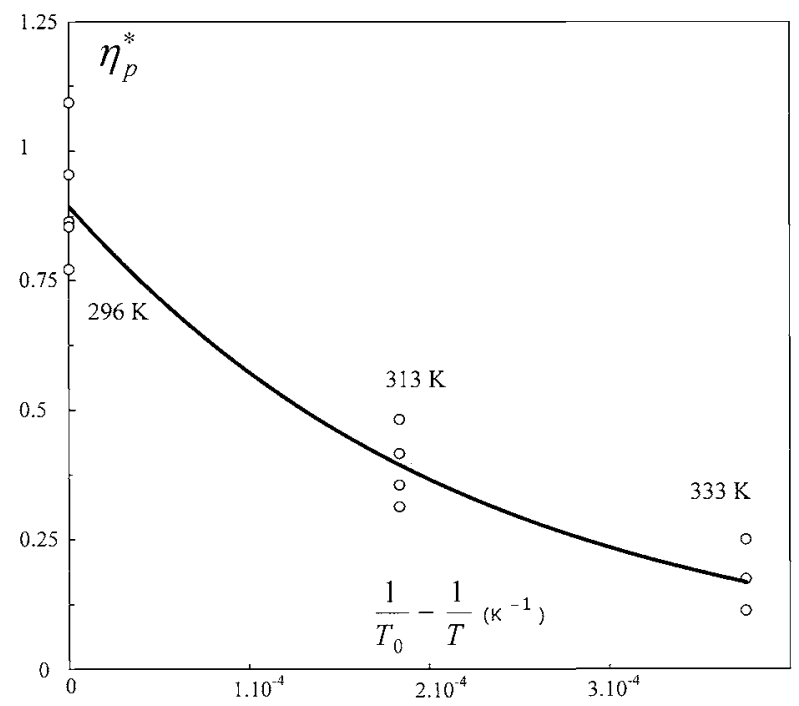

Fig. 6. Influence of temperature on $\eta_{\mathrm{p}}^{\star}$
This type of diagram enables to analyse the effect of fibre fraction by grouping in the same figure the results of each fibre fraction at every imposed strain rate. On the one hand, we note that $\eta_{\text {comp }}^{\text {smc }}$ is an increasing function of $f^{\mathrm{f}}$. On the other hand, we check that results are independent of $\dot{\varepsilon}$.

Whatever the testing temperature, result were fitted by an evolution equation similar to the one proposed by Batchelor [12] (Fig. 9):

$\eta_{\text {comp }}^{\star}=1+\beta \frac{f^{\mathrm{f}}}{1-f^{\mathrm{f}}}$

where the coefficient $\beta$, identified at each temperature, is independent of $T$ and equals 360. Finally it emerges that SMC compression viscosity can be expressed in a similar way to paste viscosity, modifying it by a function of the fibre fraction:

$\eta_{\mathrm{comp}}^{\mathrm{smc}}=3 \eta_{1}(T)\left(\dot{\varepsilon} / \dot{\varepsilon}_{0}\right)^{n^{\mathrm{s}}}\left(1+\beta \frac{f^{\mathrm{f}}}{1-f^{\mathrm{f}}}\right)$

\section{SMC shear behaviour}

To date, SMC shear behaviour has only been studied at $T_{0}$. The characteristic shape of the resulting stress/ strain curves is very similar to that of paste (Figs. 3 and 4). Just as for the latter, to analyse the effect of shear rate $\dot{\gamma}$, results were collected at a shear strain of 1 corresponding to the stress peak.

Fig. 10 shows the evolution of $\eta_{\text {shear }}^{\text {smc }}$ in terms of $\dot{\gamma}$ for a SMC containing $15.1 \%$ of fibre.

As shown in this figure, the strain rate sensitivity of the fitted power-law equals the one identified in simple compression, thus allowing to write:

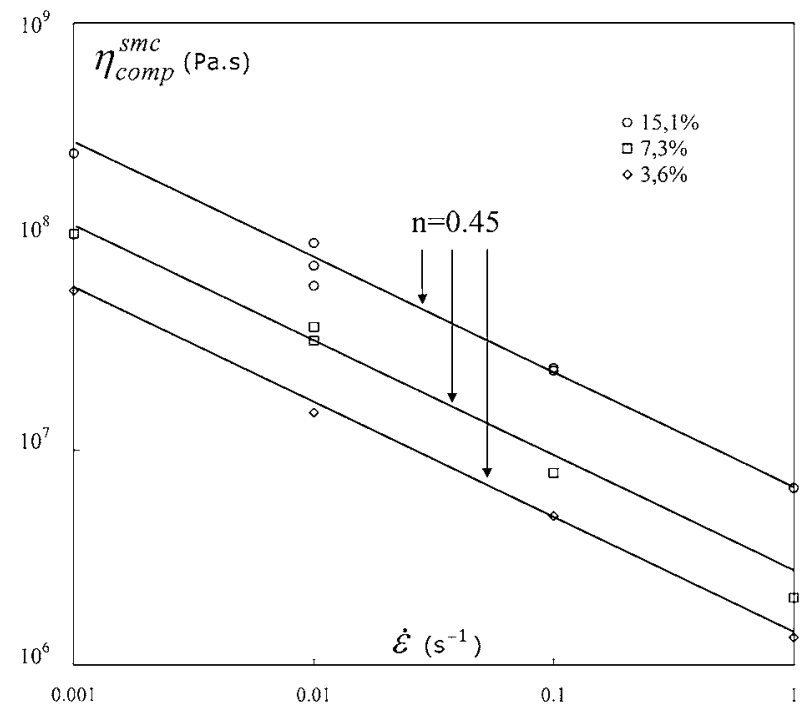

Fig. 7. Influence of $\dot{\varepsilon}$ on SMC compression viscosity at $T 296 \mathrm{~K}$ and for $f^{\mathrm{f}} \quad 3.6 \%, 7.3 \%, 15.1 \%$. 
$\eta_{\text {shear }}^{\text {smc }}=\eta_{1, \text { shear }}^{\text {smc }}\left(T, f^{\mathrm{f}}\right) \cdot\left(\frac{\dot{\gamma}}{\dot{\gamma}_{0}}\right)^{n s 1}$

Just as for compression, the effect of $f^{\mathrm{f}}$ is analysed using the reduced shear viscosity $\eta_{\text {shear }}^{\star}\left(T, f^{\mathrm{f}}\right)$ defined by:

$\eta_{\text {shear }}^{\star}=\frac{\eta_{1, \text { shear }}^{\text {smc }}\left(T_{0} f^{\mathrm{f}}\right)}{\eta_{1}\left(T_{0}\right)}$

Table 2 summarises the results obtained on the fibre fraction effect at $T_{0}$ and $\gamma=0.1 \mathrm{~s}^{-1}$.

Despite the fewer number of tests than in compression, we observe that $\eta_{\text {shear }}^{\star}$ increases far less strongly with $f^{\mathrm{f}}$ than $\eta_{\text {comp }}^{\star}$. These data, rare as yet, nevertheless let us propose a first evolution law, identified at room temperature: $\quad \eta_{\text {shear }}^{\star}\left(T_{0}, 0.1 \mathrm{~s}^{1}\right)=1+k \cdot f^{\mathrm{f}}, \quad$ where

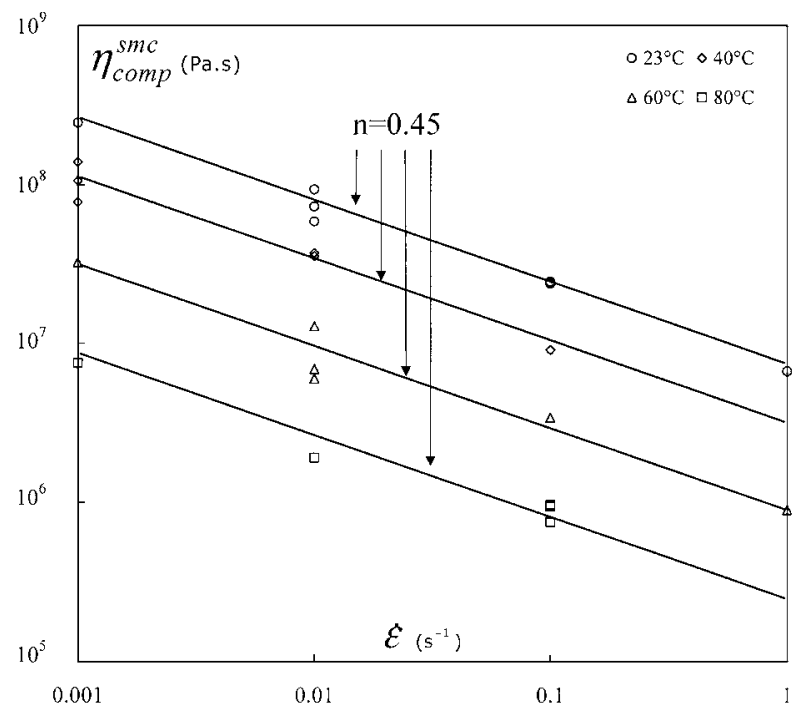

Fig. 8. Influence of $\dot{\varepsilon}$ on SMC compression viscosity at $T$ 296, 313 and $333 \mathrm{~K}$, for $f^{\mathrm{f}} \quad 15.1 \%$.

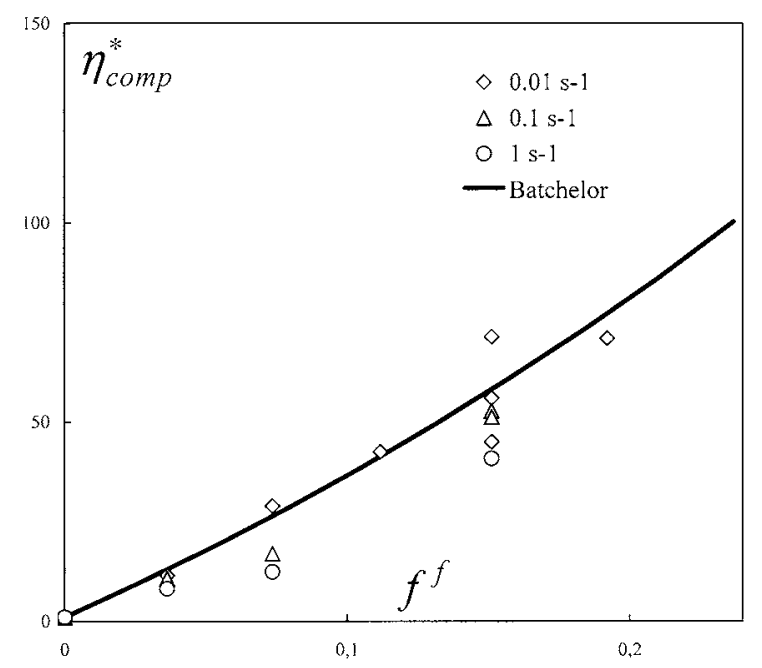

Fig. 9. Influence of fibre fraction on SMC compression viscosity. $k=18.5$. Finally, Fig. 11 gives the evolution of the ratio $\eta_{\text {shear }}^{\star} / \eta_{\text {comp }}^{\star}$ with fibre fraction. It is found that SMC anisotropy markedly increases with $f^{\mathrm{f}}$. For industrially used SMC normally containing $20 \%$ of fibre, this anisotropy ratio is around 50 .

\section{Conclusions}

The developed rheometer proved to be an adapted tool for characterising fibre-reinforced pasty media. It was shown that for strain states representative of the process, paste behaved like a Von Mises material. Its viscosity is a power-law function of strain rate and evolves according to an Arrhenius law with temperature.

The effect of fibre fraction was also studied for simple compression and simple shear. Results show that fibrereinforced SMC have a behaviour similar to that of paste, regardless of mechanical loading type and fibre fraction. It behaves like a power-law fluid with, however, a strain rate sensitivity slightly lower than that of paste. The effect of fibre fraction in simple compression was exactly identified. Nevertheless, the equation obtained for simple shear still has to be confirmed by additional tests.

However, SMC shear viscosity is less affected by the addition of fibres than their compression one so that for industrial fibre fractions, SMC behaviour is strongly

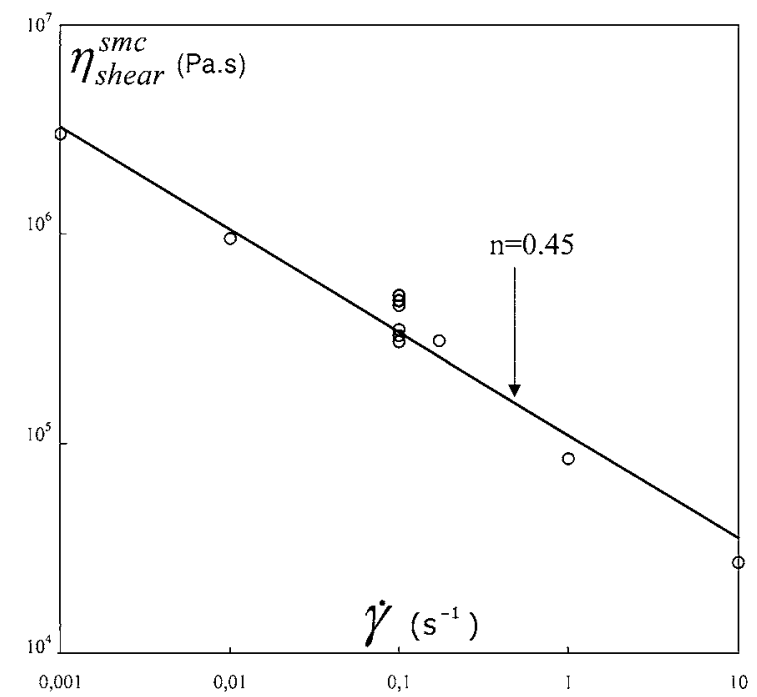

Fig. 10. Evolution of SMC shear viscosity with $\dot{\gamma}$ at $T 296 \mathrm{~K}$, $f^{\mathrm{f}} 15.1 \%$.

Table 2

Fiber fraction influence in shear, $\dot{\gamma} \quad 0.1 \mathrm{~s} \quad{ }^{1}, T \quad T_{0}$

\begin{tabular}{lllcc}
\hline$f^{\mathrm{f}}(\%)$ & 0 & 3.6 & 11.2 & 15.1 \\
$\eta_{\text {cis }}^{\star}($ Pa s $)$ & 1 & 1.98 & 2.93 & 3.47 \\
\hline
\end{tabular}




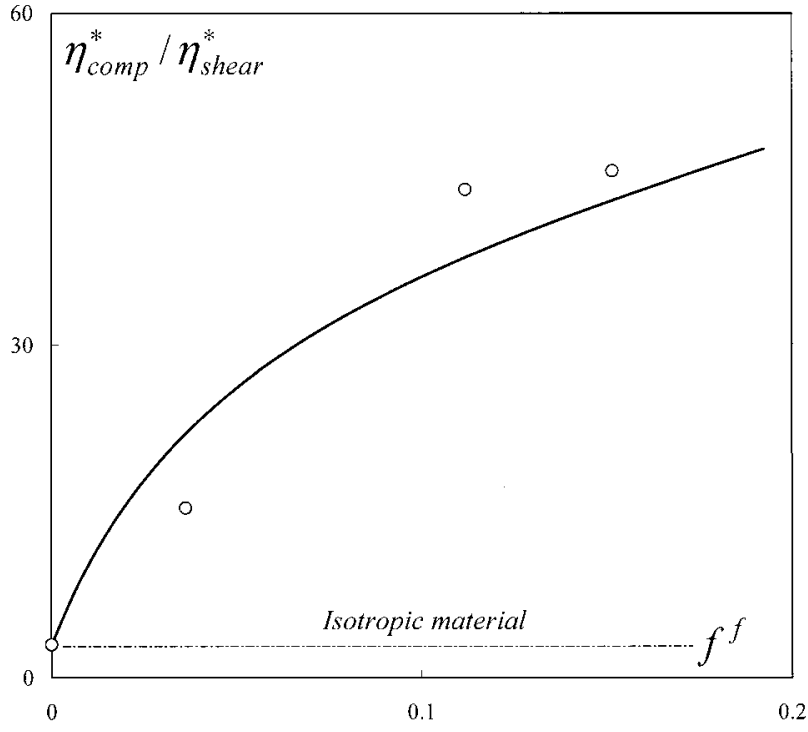

Fig. 11. Evolution of $\eta_{\text {shear }}^{\star} / \eta_{\text {comp }}^{\star}$ with the fibre fraction at room tem perature.

anisotropic. This result disables the constitutive assumptions (isotropic material) implemented in some forming simulation software [4]. To further characterise the anisotropy of SMC rheology during moulding, additional planar compression tests are required and will be carried out in a future work. These tests will enable to fully identify a more realistic transversely isotropic model of the SMC behaviour (i.e. isotropic in the plane of sheets).

\section{References}

[1] Marker LF, Ford B. Modern Plastics 1977:64 71.

[2] Barone MR, Caulk DA. Polymer Composites 1985;6(2):105 9.

[3] Silva Nieto RJ, Fisher BC, Birley AW. Polymer Composites 1980;1(1):14 23.

[4] Lee C C, Folgar F, Tucker CL. Journal of Engineering for Industry 1984;106:114 25.

[5] Barone MR, Caulk DA. Journal of Applied Mechanics 1986; 53:361 70 .

[6] Silva Nieto RJ, Fisher BC, Birley AW. Polymer Composites 1981;2(4):209 18

[7] Lee LJ, Marker LF, Griffith RM. Polymer Engineering and Science 1981;21:499 506 .

[8] Kim K T, Jeong J H, Im Y T. Journal of Material Processing Technology 1997;67:105 11.

[9] Lin C M, Weng C I, Ho C T. Polymer Composites 1997; 18(5):613 22.

[10] Servais C, Luciani A, Mansón J AE. Journal of Rheology 1999; 43(3): 100518

[11] Lin C M, Weng C I. Polymer Composites 1999;20(1):98 113.

[12] Batchelor GK. Journal of Fluid Mechanics 1971;46:545 70. 